\title{
The Universe on a Desktop: Observational Astronomy Simulations in the Instructional Laboratory
}

\author{
Laurence A. Marschall \\ Department of Physics, Gettysburg College, \\ Gettysburg, PA 17325, USA \\ marschal@gettysburg.edu
}

Received 1999 December 1, accepted 2000 March 15

\begin{abstract}
Though the value of hands-on learning has long been recognised by educators, it is difficult to design laboratories in astronomy classes that present realistic astrophysical techniques to undergraduate students. Unlike most other sciences, astronomy is largely observational, not experimental, and making useful observations involves expensive equipment over time scales inconvenient for pedagogy. In recent years, however, astronomy has gone almost completely digital, and the advent of large on-line databases and fast personal computers has made it possible to realistically simulate the experience of research astrophysics in the laboratory. Since 1992, Project CLEA (Contemporary Laboratory Experiences in Astronomy) has been developing computer-based exercises aimed primarily at the introductory astronomy laboratory. These exercises simulate important techniques of astronomical research using digital data and Windows-based software. Each of the nine exercises developed to date consists of software, technical guides for teachers, and student manuals for the exercises. CLEA software is used at many institutions in all the United States and over 60 countries worldwide, in a variety of settings from middle school to upper-class astronomy classes. The current design philosophy and goals of Project CLEA are discussed along with plans for future development.
\end{abstract}

Keywords: astronomy education—laboratories—software

\section{On the Difficulty of providing Hands-on Exercises in Astronomy}

Educational research in recent years has affirmed the common perception that experience is the best teacher, especially in introductory science courses (Hake 1998). Hands-on activities, usually in a laboratory setting, help students learn through several processes. In the first place, experiential work helps clarify concepts by compelling students to apply their understanding of a subject to a concrete situation. If all works as expected, the student's confidence grows, and the student is motivated by an increasing sense of mastery. Even if an experiment 'fails', that is, if it does something strange or unexpected, a student may be forced to rethink preconceived notions, and thereby gain a firmer understanding of what might otherwise have been shaky misconceptions.

But astronomers have never been as successful as other scientists in providing effective exercises for the introductory laboratory. Part of the problem, of course, is that astronomy is observational, rather than experimental. Observing at night is the least of the problem. A simple measurement of the height of a lunar mountain requires observing the shadow of the mountain at a particular phase of the moon, one or two nights, perhaps, of a month. If those nights are cloudy, the observing exercise is an exercise in futility. Or consider the measurement of the light curve of an eclipsing binary star. If the period of the star is several days, it may take weeks, or even months, to sample the entire cycle of variations. Complete phase coverage is particularly difficult if the period of variation is close to an integral number of days.

Astronomy teachers have addressed these difficulties, for the most part, by providing students with predigested data: images taken by professional astronomers, spectra from observatories, and tables of reduced data from journal articles. In these 'dry lab' exercises, students measure the photographs with magnifying glasses and rulers, or transfer the tabulated data onto a graph and answer a few questions about the result (Bruck 1990; Culver 1984; Ferguson 1990; Hoff, Kelsey \& Neff 1992; Johnson \& Canterna 1987). Not surprisingly, many students view such labs as little more than clerical work, an activity designed to fill up a few hours, with very little excitement and even less intellectual content. But what else are astronomers to do?

The development of digital astronomy in the 1980s provided an attractive solution to this problem. By the end of the decade, astronomical observation was regularly performed using computer-controlled instruments, data were stored in digital format, and data reduction was invariably carried out on a computer. As a result, it became possible to simulate real observations with surprising fidelity using desktop PCs. Actual data from on-line archives could be used, making it possible for simulation programs to incorporate the look and feel of real-time telescopes, and to produce results as authentic as those from a professional observatory. Students could now conveniently and effectively obtain a grounding in the basic techniques and strategies of modern astronomy. 
The growing availability of simulations in the astronomy laboratory was a natural outgrowth of the computer revolution of the 1980 s, but the use of simulations to teach technical subjects was not unprecedented. Aircraft pilots have been trained on simulators for almost half a century. Simulations are also used widely for training in certain medical fields, like endoscopic surgery, where remote sensing and remote manipulation were standard operating-room procedures. The attraction of simulations in these fields, as in astronomy, was the ability to effectively impart a hands-on experience without the risk or expense of a real-world exercise.

\section{Project CLEA: Digital Simulations in the Introductory Laboratory}

In the early 1990s, a major project to develop a suite of introductory laboratory simulations in astronomy, Contemporary Laboratory Experiences in AstronomyProject CLEA, was funded by the National Science Foundation. Inspired by earlier simulations by John Trasco at the University of Maryland and Michael Seeds at Franklin and Marshall College, Project CLEA aimed at producing exercises with two principal goals in mind: to reinforce important concepts in astronomy, and to help students experience how astronomy is done (Marschall 1998). While the project aimed to institute a major reform in astronomy education, its tactics were not to design a totally integrated curriculum. Recognising that teachers would pick and choose from the curriculum it developed, CLEA designed its packages to be modular, self-contained exercises, each containing a computer program, a student manual, and a technical teacher's guide. The labs were not tied to any particular text, but it was assumed that they would supplement, rather than replace, the standard classroom lecture course. If demographics are any indication, this approach has been quite successful. CLEA material, distributed freely through the web at www.gettysburg.edu/academics/physics/clea/ CLEAhome. html, and by mail, have been adopted by institutions in all 50 states and more than 60 nations worldwide. We are aware of CLEA materials that have been translated into Spanish, Dutch, Italian, Hebrew, and Polish. CLEA exercises are used to teach practical astronomy at a number of Australian institutions, including the University of Sydney, the University of Western Sydney, and Swinburne University of Technology. While exact numbers of classroom users worldwide are difficult to gauge, an informal survey of the user database indicates that the number of students using CLEA exercises yearly is in the tens of thousands.

\section{Design Philosophy of the Project}

Though they cover a wide range of topics, from Kepler's laws to cosmology, the CLEA exercises have been developed with a unified design philosophy in mind:
(1) We try not to do with computers that which can be done equally well with real experiments. The best way to learn the constellations, we believe, is to observe outdoors. Exercises using laboratory spectra, or illustrating geometrical optics and telescopes, are best done using real gas discharge tubes, lenses, and mirrors. On the other hand exercises involving spectroscopy or photometry of faint stars, or radio observations of pulsars, can be better presented as simulations.

(2) Software is designed with flexible user-defined options that make it useful both for introductory non-science majors and upper-class science students. Default settings, however, are for the introductory students who make up the majority of our users.

(3) Student workbooks are provided for introductory non-science students. Learning objectives and goals are stated explicitly in these workbooks, and the exercises are designed to take into account the results of cognitive research in physics learning (see e.g. Arons 1990).

(4) Exercises avoid, as much as possible, rote copying and graphing. They emphasise how data are collected and analysed, or demonstrate phenomena that cannot be examined otherwise.

(5) Exercises include instructive 'real-life' features, such as detector noise, sky background, and weather interruptions, while avoiding complications that might distract from the principal learning objectives of the lab.

(6) Exercises are carefully designed for ease of use and uniformity. Extensive feedback from both students and faculty at other institutions makes the software easy-to-use and highly portable. This adds greatly to development time, but it insures that the materials we produce will have lasting value to a wide range of users.

\section{Details of the Exercises}

There are currently nine CLEA exercises, covering a wide range of techniques and concepts from planetary astronomy to cosmology. The software runs under Windows, although one colour Mac lab is available, and a number of rudimentary black and white Mac programs, lacking in many of the features of the Windows programs, are available as well. A brief description of each exercise follows:

(1) Revolution of the moons of Jupiter. Students observe the moons of Jupiter at fixed intervals for several weeks to verify Kepler's third law and to measure the mass of Jupiter.

(2) Rotation of Mercury using Doppler radar. Students measure the equatorial velocity of Mercury using radar echoes from a simulated radio telescope. 
(3) Flow of energy out of the Sun. Students conduct experiments in radiative transfer of photons from the core of the Sun to the surface (illustrating random walk), and through the atmosphere (illustrating the formation of absorption and emission lines).

(4) Classification of stellar spectra. Students practice classifying digital stellar spectra using an atlas of digitised spectra and unknowns. They then use a simulated optical telescope with a photon-counting spectrometer to obtain spectra of stars from a simulated star field, and by classifying the spectra they obtain the distance to the stars.

(5) Photoelectric photometry of the Pleiades. Students use a photon-counting photometer attached to a simulated telescope to obtain B and $\mathrm{V}$ magnitudes of stars in the Pleiades. They use their data to construct an HR diagram of the cluster and to determine the distance of the cluster.

(6) Hubble redshift distance relation. Students use a photon-counting spectrometer attached to a simulated telescope to obtain spectra of galaxies in five galaxy clusters at various distances from the Milky Way. By measuring the redshifts of prominent spectral features they plot a velocitydistance graph, determine the Hubble constant, and calculate the expansion age of the Universe.

(7) Large-scale structure of the Universe. Students use a photon-counting spectrometer attached to a simulated telescope to obtain spectra of galaxies in a sample chosen from published large-scale surveys of a slice of nearby space. By measuring the redshifts of prominent spectral features they plot a 'wedge diagram' showing the three-dimensional distribution of the galaxies, including the 'Great Wall'. Students can pool data gathered collaboratively using network software.

(8) Radio astronomy of pulsars. Students use a simulated steerable radio telescope to investigate the properties of pulsars. They measure period and amplitude of selected pulsars as a function of frequency. They then use multi-frequency observations to determine the distance of pulsars from the dispersion in arrival times of pulses.

(9) Astrometry of asteroids. Students use a specialised image analysis program to blink pairs of images taken with a research telescope. They find asteroids as moving objects, measure the coordinates of the objects by using a digital catalog of reference stars (the Hubble Guide Star Catalog), and determine the angular velocity of the asteroid. They also measure the coordinates of the asteroid on two images taken simultaneously from sites several thousand $\mathrm{km}$ apart, and use parallax to determine the distance and velocity of the asteroid (Ratcliff et al. 1993).
A research version of the software used for the astrometry exercise, called Toolkit for Astrometry, has also been developed. The software makes it easy to plan observations of asteroids, blink and measure large images from professional telescopes, and produce ephemerides and finding charts.

\section{Prospects for Future Development}

Project CLEAs plans for the next two years include the development of three more exercises. The one under current development is called The Search for Object X, which is meant to be a capstone exercise for students who have previously performed other CLEA exercises. Like a chemistry laboratory exercise in qualitative analysis, where students are asked to determine the composition of a white powder, the 'Object X' lab asks students to determine the nature of an astronomical object for which only the coordinates are specified. They can use a CCD, photometer, spectrometer, or radio telescope to investigate the 'white blob' that is their object, using techniques and information learned from earlier CLEA labs to analyse their observations and draw a reasoned conclusion from the data.

Two other exercises planned for further development Include: (1) an exercise on radial velocity measurements of binary stars and the search for extrasolar planets; and (2) an exercise on variable stars and the Cepheid distance scale. With the completion of these exercises, CLEA will have available an even dozen laboratory experiments covering many of the major techniques of modern astronomy.

We plan to continue developing our exercises for the Windows platform, but there is a growing interest among our users in educational software which can be run on browsers and actually run over the web. Though there is some astronomy software already available in Java, we have not yet found the web amenable to the highly interactive demands of our programs. But we are studying the situation closely, and intend to develop some prototypes of web-based exercises within the next two years. As the capabilities of both computers and the internet grow, we will doubtless see the development of some exciting new applications of simulations for the astronomy laboratory.

\section{Acknowledgments}

Thanks go first and foremost to my co-investigators at Project CLEA, Glenn Snyder, Rhonda Good, Mike Hayden, Dick Cooper and Mia Luehrmann; to our evaluators, Marcus Lieberman and Gina Brissenden; and to the many astronomers and teachers around the world who have offered criticism and encouragement. Thanks most of all to the students who have used our materials and who have taken additional time to provide feedback on how they liked it and how well it was working. 
Project CLEA has been supported by grants from the National Science Foundation and Gettysburg College. CLEA software is available free of charge from www.gettysburg.edu/academics/physics/clea/ CLEAhome.html.

\section{References}

Arons, A. A. 1990, A Guide to Introductory Physics Teaching (New York: Wiley)

Bruck, M. T. 1990, Exercises in Practical Astronomy Using Photographs (Bristol: Adam Hilger)
Culver, R. 1984, An Introduction to Experimental Astronomy (San Francisco: W. H. Freeman)

Ferguson, D. C. 1990, Introductory Astronomy Exercises (Belmont, Calfornia: Wadsworth)

Hake, R. R. 1998, Am. J. Phys., 66, 64

Hoff, D. B., Kelsey, L. J., and Neff, J. S. 1992, Activities in Astronomy, 3rd edn (Dubaque, Iowa: Kendall-Hunt)

Johnson, P. E., and Canterna, R. 1987, Laboratory Experiments for Astronomy (New York: Saunders College Publishing)

Marschall, L. A. 1998, in New Trends in Astronomy Teaching, ed. L. Gouguenheim et al. (Cambridge Univ. Press), pp. 79-87

Ratcliff, S. J., Balonek, T. J., Marschall, L. A., DuPuy, D. L, Pennypacker, C. R., Verma, R., Alexov, A., and Bonney, V. 1993, Am. J. Phys., 61, 208 